Research Paper

\title{
Triterpenoid Saponin W3 from Anemone flaccida Suppresses Osteoclast Differentiation through Inhibiting Activation of MAPKs and NF-KB Pathways
}

\author{
Xiangying Kong ${ }^{1}$, Yue Yang ${ }^{1}$, Wenbin $\mathrm{Wu}^{1}$, Hongye Wann ${ }^{1}$, Xiaomin $\mathrm{Li}^{1}$, Michun Zhong ${ }^{1}$, Xiaohui Su${ }^{1}$, \\ Shiwei Jia ${ }^{2}, \mathrm{Na} \operatorname{Lin}^{1} \bowtie$ \\ 1. Institute of Chinese Materia Medica, China Academy of Chinese Medical Sciences, 100700 Beijing, China. \\ 2. Guangzhou Kanghe Pharmaceutical Limited Company, 511440 Guangzhou, China
}

$\square$ Corresponding author: Na Lin. Institute of Chinese Materia Medica, China Academy of Chinese Medical Sciences, No. 16, Nanxiaojie, Dongzhimennei, Beijing 100700, China. Tel:+861064014411-2869; Fax: +861064013996. E-mail: linna888@163.com

(C) 2015 Ivyspring International Publisher. Reproduction is permitted for personal, noncommercial use, provided that the article is in whole, unmodified, and properly cited. See http://ivyspring.com/terms for terms and conditions.

Received: 2015.04.01; Accepted: 2015.07.17; Published: 2015.08.15

\begin{abstract}
Excessive bone resorption by osteoclasts within inflamed joints is the most specific hallmark of rheumatoid arthritis. A. flaccida has long been used for the treatment of arthritis in folk medicine of China; however, the active ingredients responsible for the anti-arthritis effects of $A$. flaccida are still elusive. In this study, W3, a saponin isolated from the extract of $A$. flaccida was identified as the major active ingredient by using an osteoclast formation model induced by receptor activator of nuclear factor kappa-B ligand (RANKL). W3 dose-dependently suppressed the actin ring formation and lacunar resorption. Mechanistic investigation revealed that W3 inhibited the RANKL-induced TRAF6 expression, decreased phosphorylation of mitogen-activated protein kinases (MAPKs) and IKB- $\alpha$, and suppressed NF- $\mathrm{KB}$ p65 DNA binding activity. Furthermore, W3 almost abrogated the expression of c-Fos and nuclear factor of activated T cells (NFATcl). Therefore, our results suggest that W3 is a potential agent for treating lytic bone diseases although further evaluation in vivo and in clinical trials is needed.
\end{abstract}

Key words: Anemone flaccida; RANKL; Osteoclasts; Bone resorption

\section{Introduction}

Osteoblast-induced bone formation and osteoclast-mediated bone resorption are the major events that contribute to bone homeostasis. Disrupting the equilibrium of osteoblasts and osteoclasts in their differentiation, activation and survival may result in various skeletal disorders. It has been well established that osteoclasts derived from the monocyte-macrophage lineage are the primary cells responsible for bone resorption. Thus altered osteoclast formation and bone resorption are usually the pathological feature of several lytic bone diseases, such as osteoporosis and rheumatoid arthritis (RA) [1, 2]. Therefore, osteoclast becomes the main target of current anti-resorptive drugs.
The dry root of Anemone flaccida Fr. Schmidt (Di $\mathrm{Wu}$ in Chinese), is widely used in clinical compound prescription for the treatment of Rheumatic diseases, external wounds and inflammations in China. Saponins are the characteristic components and also the main active ingredients of $A$. flaccida [3]. Among other biological activities, total saponins (TS) isolated from A. flaccida possess anti-inflammation, immunoregulatory and analgesia properties [4]. Triterpenoid saponin W3, a natural occurring saponin, is the major active component and the highest content of the TS found in A. flaccida, also can be synthesized chemically in vitro [5]. Previous reports showed that W3 exerts antitumor effect via the COX-2/ $\mathrm{PGE}_{2}$ pathway in vitro 
[6], and also regulates immune activity in mice [4]. Interestingly, W3 showed similar effect as TS in treatment of RA [7]. Focal bone destruction within inflamed joints is the most specific hallmark of RA and leads to deformation, laxity, and functional disability [8]. However, to date the effect of W3 on bone metabolism is not clear.

In the present study, we investigated the direct effect of W3 on osteoclast differentiation in receptor activator of nuclear factor kappa-B ligand (RANKL)-induced RAW 264.7 cells. Moreover, the possible mechanism associated with its inhibitory effect on osteoclast differentiation was also explored.

\section{Materials and Methods}

\section{Chemicals}

Alpha Modified Eagles Medium (a-MEM), fetal bovine serum (FBS), penicillin and streptomycin, were purchased from Invitrogen Life Technologies (NY, USA). Mouse RANKL was obtained from PeproTech (Rocky Hill, NJ, USA). Antibodies against NFATc1 and TRAF6 were purchased from Santa Cruz Biotechnology (Santa Cruz, CA, USA). Antibodies against c-Fos, p-ERK, ERK, p-p38, p38, p-JNK, JNK and GAPDH were obtained from Cell Signaling Technology (Beverly, MA, USA). NE-PER Nuclear and Cytoplasmic Extraction Reagent Kit and LightShift ${ }^{\mathrm{TM}}$ Chemiluminescent EMSA Kit was provided by Pierce Biotechnology (Rockford, IL, USA).

\section{Preparation of Total Saponins and W3}

Rhizome of A. flaccida was collected from Jiufeng County of Hubei Province, China. Roots of Anemone flaccida $(1.0 \mathrm{~kg})$ were extracted twice with $10 \mathrm{~L}$ water for $1.5 \mathrm{~h}$. The extracting solution was filtered and concentrated under reduced pressure to proper amount. Then it was deposited by adding ethanol to make the whole solution into $75 \%$ ethanol solution. Overnight, the supernatant liquor was filtered and condensed under reduced pressure. The residue was dissolved in water, and load on a glass column $(10 \times 150 \mathrm{~cm})$ containing $3000 \mathrm{~g}$ D101 macroporous resin. First, water was used to wash the unabsorbed substances until the eluted solution became nearly colorless. Then $70 \%$ ethanol $(\mathrm{v} / \mathrm{v})$ was selected to elute the column. The solution was evaporated to dryness under reduced pressure, and then it was subjected to a low pressure reversed-phase $\mathrm{C} 18$ chromatography column using $\mathrm{CH}_{3} \mathrm{OH}: \mathrm{H}_{2} \mathrm{O}$ (70:30) to obtain fraction I and II . Fraction II were collected and decompressed to yield $35.38 \mathrm{~g}$ residue (dried weight), which was considered as TS.

The quantitative determination of TS was performed on by Agilent 1160 HPLC system (Agilent
Technologies, CA, USA). The sample was separated on a Hydrosphere C18 column $(150 \times 2.1 \mathrm{~mm}, 3 \mu \mathrm{m})$ with the oven temperature maintained at $35^{\circ} \mathrm{C}$. $\mathrm{CH}_{3} \mathrm{OH}: \mathrm{H}_{2} \mathrm{O}(70: 30$, v/v) was used as mobile phase for the LC separation. The flow rate was at 0.2 $\mathrm{mL} / \mathrm{min}$ and peaks were detected at $205 \mathrm{~nm}$. An aliquot of $5 \mu \mathrm{L}$ of TS solution was injected into the HPLC system for analysis.

\section{Cell Culture}

RAW 264.7 cells (ATCC, Manassas, VA, USA) were grown in a-MEM supplemented with $10 \%$ heat inactivated FBS, $2 \mathrm{mM}$ L-glutamine and $100 \mathrm{U} / \mathrm{mL}$ penicillin/streptomycin. Incubations were performed at $37^{\circ} \mathrm{C}$ in $5 \% \mathrm{CO}_{2}$, and cultures fed every $2-3$ days by replacing with fresh medium. Bone marrow-derived macrophages (BMMs) were collected from tibia and femur of 4-7-week-old SD rat by flushing the marrow space with a-MEM. After removing the red blood cells (RBCs) with ACK buffer (0.01 mM EDTA, $0.011 \mathrm{M}$ $\mathrm{KHCO}_{3}$, and $0.155 \mathrm{M} \mathrm{NH}_{4} \mathrm{Cl}, \mathrm{pH} 7.3$ ), cells were cultured for 1 day in a-MEM containing 10\% fetal bovine serum (FBS). Non-adherent cells were collected and further cultured with $20 \mathrm{ng} / \mathrm{mL}$ M-CSF in a-MEM containing $10 \%$ FBS. After 3-4 days, culture medium was removed and adherent cells (BMMs) were used for osteoclast differentiation.

\section{Enzyme Linked Immunosorbent Assay (ELISA)}

RAW 264.7 cells were suspended in a-MEM containing $10 \%$ FBS and seeded at a concentration of $1 \times 10^{5}$ cells/well to a 24 -well culture plate, followed by stimulated with RANKL (50 ng/mL) in the presence or absence of TS, W1, W2, W3 or Saponin F for $24 \mathrm{~h}$. At the end of culture, medium was collected and analyzed for TNF- $\alpha$ using the ELISA kit (R\&D system, USA) according to the manufacturer's instructions.

\section{Osteoclast Formation}

RAW 264.7 cells or BMMs were seeded onto a 96 well plate $\left(1 \times 10^{4}\right.$ cells/well $)$ with complete a-MEM containing RANKL (50 ng/mL) and $20 \mathrm{ng} / \mathrm{mL}$ M-CSF in the presence of TS, W1, W2, W3 or Saponin F or the vehicle control for 6 days at $37^{\circ} \mathrm{C}$ and $5 \% \mathrm{CO}_{2}$. The experiments were carried out 3 times in triplicate measurements.

\section{TRAP Staining}

After 6 days of osteoclast formation as described above, cells were washed with PBS and fixed with $4 \%$ paraformaldehyde for $15 \mathrm{~min}$. Fixed cells were subjected to an assay for Tartrate-resistant acid phosphatase (TRAP) activity according to the manufacturer's protocol (Sigma Alrich, St Louis, MO, USA). The images were taken with a digital camera attached 
to the microscope. TRAP positive multinucleated cells (>3 nuclei) were scored as osteoclast-like (OCL) cells. The number of TRAP-positive cells was counted using an eyepiece graticule at a magnification of 100 and the results expressed as the number of cells per visual field.

\section{Bone Resorption Pit Assay}

To study the effect on bone resorption, RAW 264.7 cells were seeded onto $50 \mu \mathrm{m}$ thick bovine bone slices and incubated with a-MEM containing RANKL (50 ng/mL) in the presence of W3 or the vehicle control for 7 days at $37^{\circ} \mathrm{C}$ and $5 \% \mathrm{CO}_{2}$. After 7 days, cells were removed by sonication and the bovine bone slices was stained to identify resorption pits with toluidine blue staining. Resorption lacunaes were also visualized using a Hitachi S-3400N scanning electron microscope (Hitachi High-tech, Japan).

\section{Immunofluorescent Staining}

For immunofluorescence studies, RAW 264.7 cells were stimulated with RANKL ( $50 \mathrm{ng} / \mathrm{mL})$ for 6 days to induce osteoclast formation in the presence or absence of W3 $(0.1,0.5$ and $2.5 \mu \mathrm{g} / \mathrm{mL})$. After incubation, the cultures were fixed in $4 \%$ paraformaldehyde in PBS and cells permeabilized with $0.1 \%$ Triton X-100 in PBS for 15 min. Cells were then stained for F-actin by incubation in TRITC-conjugated phalloidin (Sigma Alrich, St Louis, USA) for $45 \mathrm{~min}$ at $37^{\circ} \mathrm{C}$, then washed with PBS and mounted with Hoechst 33258 (Vector Laboratories, Peterborough, UK). Cells were inspected with an Olympus $x 41$ microscope. The experiments were carried out 3 times in triplicate measurements.

\section{Cell Survival Viability Assay}

Cell viability was determined by 3-(4,5-dimethyl-2-thiazolyl)-2,5-diphenyl-2H-tetrazoli um bromide (MTS) method using CellTiter $96^{\circledR}$ AQueous One Solution Cell Proliferation Assay from Promega (Madison, WI, USA) according to the manufacturer's instructions. The experiments were carried out 3 times in triplicate measurements.

\section{Western Blotting}

RAW 264.7 cells were cultured with complete a-MEM containing RANKL $(50 \mathrm{ng} / \mathrm{mL})$ in the presence of $\mathrm{W} 3$ or the vehicle control for indicated time at $37^{\circ} \mathrm{C}$ and $5 \% \mathrm{CO}_{2}$. Western blot was performed according to our previously described protocol [9]. The primary antibodies included antibody for both total and phosphorylation of ERK1/2, JNK, p38, anti-p-IkBa, anti-c-Fos, anti-TRAF6 and anti-GAPDH. Horseradish peroxidase (HRP)-conjugated anti-mouse and anti-rabbit antibodies were used as secondary antibodies. The membranes were then visualized using the ECL system. GAPDH (internal control) was used to confirm equal protein loading. The experiments were carried out 3 times in triplicate measurements.

\section{Electrophoretic Mobility Shift Assay (EMSA)}

RAW 264.7 cells were incubated with or without various concentrations of $\mathrm{W} 3(0.1,0.5$ and $2.5 \mu \mathrm{g} / \mathrm{mL})$ for $12 \mathrm{~h}$, then RANKL ( $50 \mathrm{ng} / \mathrm{mL}$ ) were added for 30 $\mathrm{min}$. The nuclear extracts were prepared by a NE-PER Nuclear and Cytoplasmic Extraction Reagent Kit. The LightShiftTM Chemiluminescent EMSA Kit and synthetic biotin labeled double-stranded NF-кB consensus oligonucleotide (5'-AGTTGAGGGGACTTTCC CAGGC-3 ${ }^{\prime}$ ) were used to measure the effect of the W3 on NF- $\mathrm{BB}$ nuclear protein-DNA binding activity according to the manufacturer's instructions. The DNA/protein complex was electrophoresed on $5 \%$ non-denaturing polyacrylamide gel. The DNA mobility shift due to binding of NF- $\mathrm{kB}$ complex was detected with enhanced chemiluminescence (ECL) reagent.

\section{Statistical Analysis}

All raw data were processed by authorized software SPSS 13.0. The data were analyzed by One-way ANOVA followed by Dunnett's t-test to assess the statistical significance of the differences between the study groups. Differences were considered statistically significant when $P$ was less than 0.05 .

\section{Results}

\section{Structure and percent composition}

TS were isolated from A. flaccidas, and the relative contents of single triterpenoid saponins are detected and determined by a normalization method of chromatographic peak areas. As shown in Fig 1, there are mainly five single triterpenoid saponins included in the TS: named W1, Saponin R, Saponin F , W2 and W3, which is from peak 1-5 in order, and the structure of these single saponin were provided in Figure 1. Among them, W3 is the most abundant saponin, reaching up to $51.8 \%(\mathrm{w} / \mathrm{w})$ in TS.

\section{W3 attenuates RANKL-induced osteoclast differentiation}

Since RAW 264.7 cells differentiate into osteoclast-like (OCL) cells as described elsewhere [10], the effect of TS and the single triterpenoid saponin on RANKL-induced osteoclast formation was examined by this model. RAW 264.7 cells cultured in the presence of RANKL formed multinucleated TRAP-positive OCL cells (Fig. 2A). When treated with TS, W1, W2, W3 and Saponin F $(0.1,0.5$ and 2.5 $\mu \mathrm{g} / \mathrm{mL}$ ) in RAW 264.7 cells, showed inhibition of os- 
teoclast formation with different degree as measured by counting the number of TRAP positive multinucleated cells. W3 inhibited the TRAP positive multinucleated cells most effectively, followed by W2, whereas W1 and saponin F to a lesser extent. TS were used as a positive control and significantly inhibited the RANKL-induced TRAP-positive OCL cells (Fig. 2

A

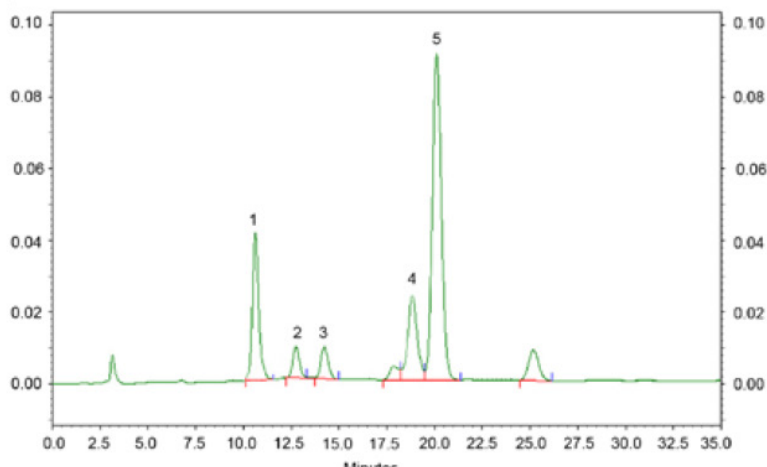

$A$ and B). Interestingly, OCL cells in cultures that were treated with TS and the single triterpenoid saponin exhibited morphological differences with control OCL cells, being smaller in size and containing fewer numbers of nuclei compared to osteoclasts in control (Fig 2A).

\section{B}

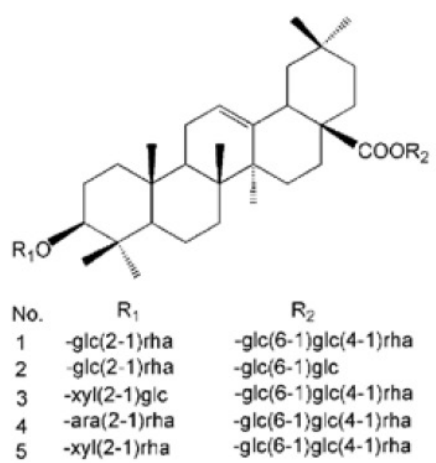

Fig. 1. Analysis of the phytochemical profile of TS. (A) Phytochemical characteristics of TS were analysed by high performance liquid chromatography (HPLC). (B) Structures of the triterpenoid saponin examined. 1: W1; 2: Saponin R; 3: Saponin F; 4: W2; and 5: W3.

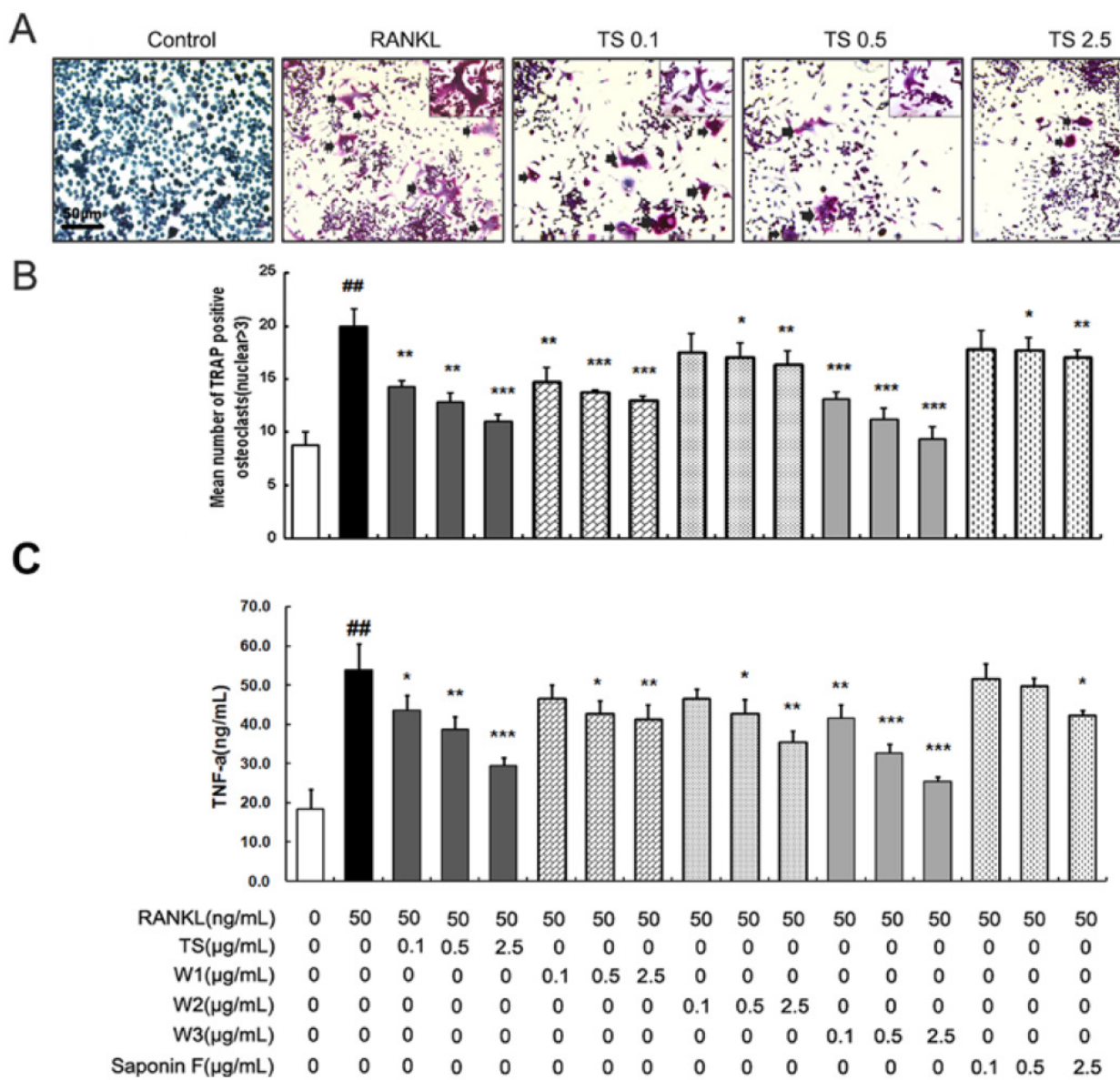

Fig. 2. TS and its triterpenoid saponin constituents inhibit RANKL-induced osteoclast differentiation. (A) RAW 264.7 cells were treated with different concentrations of TS and its triterpenoid saponin constituents $(0.1,0.5$ and $2.5 \mu \mathrm{g} / \mathrm{mL})$ in the presence of RANKL. Six days post-culture, cells were fixed with $4 \%$ paraformadehyde followed by TRAP staining. Representative images of TRAP staining of osteoclast from one of the three experiments are shown. (B) Quantitative analysis shows the mean number of TRAP-positive osteoclast. (C) Confluent RAW264.7 cells were pretreated with TS and its triterpenoid saponin constituents ( $0.1,0.5$ and $2.5 \mu \mathrm{g} / \mathrm{mL}$ ) or solvent for $2 \mathrm{~h}$, then induced with RANKL for another $24 \mathrm{~h}$. The supernatants were collected and analyzed for TNF- $\alpha$ by enzyme-linked immunosorbnent assay (ELISA). All bar graphs represent mean \pm SD of three independent experiments. $\# P<0.001$ significantly different from Control. $* P<0.05$, $* * P<0.01$ and $* * * P<0.001$ significantly different from RANKL only group. 
Inflammatory cytokines, such as TNF- $\alpha$, induces osteoclasts formation, and stimulates osteoclastic bone resorption [11]. To screen the effect of TS and the triterpenoid saponins on osteoclasts, the production of TNF- $\alpha$ induced by RANKL was studied on RAW 264.7 cells in vitro. As shown in Fig. 2C, RAW 264.7 cells produced a large quantity of TNF- $\alpha$ in response to RANKL stimulation. Treatment with TS, W1, W2, W3 and saponin F (0.1, 0.5 and $2.5 \mu \mathrm{g} / \mathrm{mL})$ could suppress TNF- $\alpha$ production in different extent, which is similar to the tendency on TRAP-positive cell number. To exclude the possibility that the observed inhibitory effect on osteoclast differentiation maybe due to cytotoxicity, we examined the survival rates of TS, W1, W2, W3 and Saponin F alone or in combination with RANKL on RAW 264.7 cells after $48 \mathrm{~h}$ treatment using MTS assay. TS and its triterpenoid saponins constituents up to $100 \mu \mathrm{g} / \mathrm{mL}$ showed no toxic to the cells (data not shown).

\section{W3 suppresses RANKL-stimulated osteoclas- tic bone resorption and $F$-actin rings formation}

To examine the effects of W3 on osteoclast formation and osteoclastic bone resorption, TRAP staining, toluidine blue staining or scanning electron microscopy were visualized and scored. Treatment of W3 resulted in reduced OCLs numbers and pit areas, or very shallower resorption pits as compared to control both by toluidine blue staining or scanning electron microscopy, which show the consistent tendency with the effect of W3 on osteoclast formation (Fig. 3).

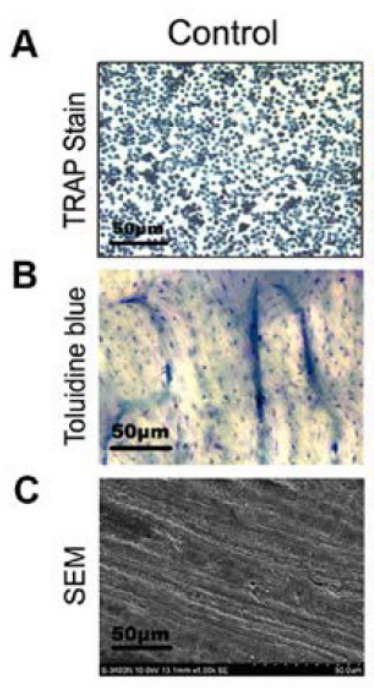

D

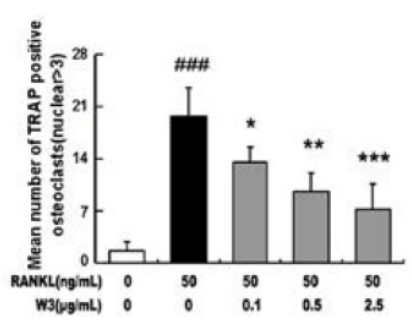

RANKL
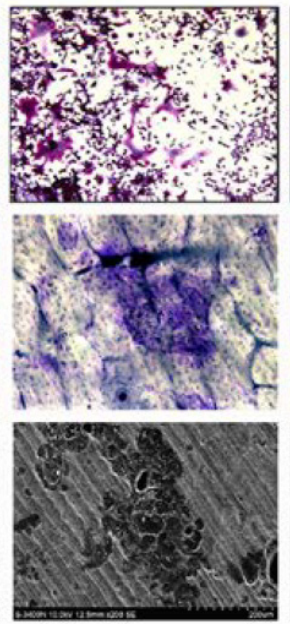

E

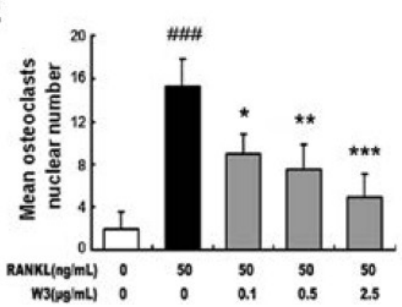

W3 0.5
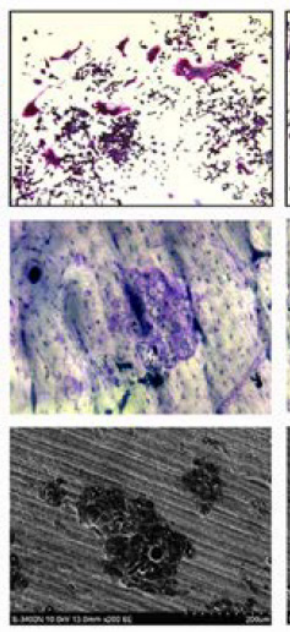

H

F

H

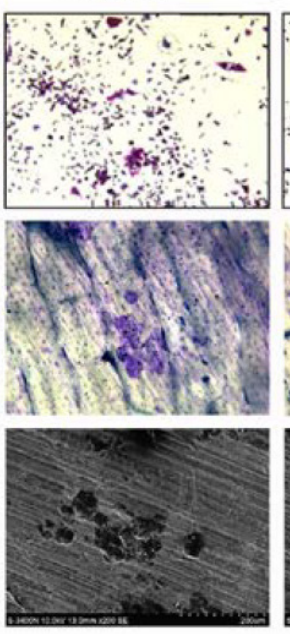

W3 2.5
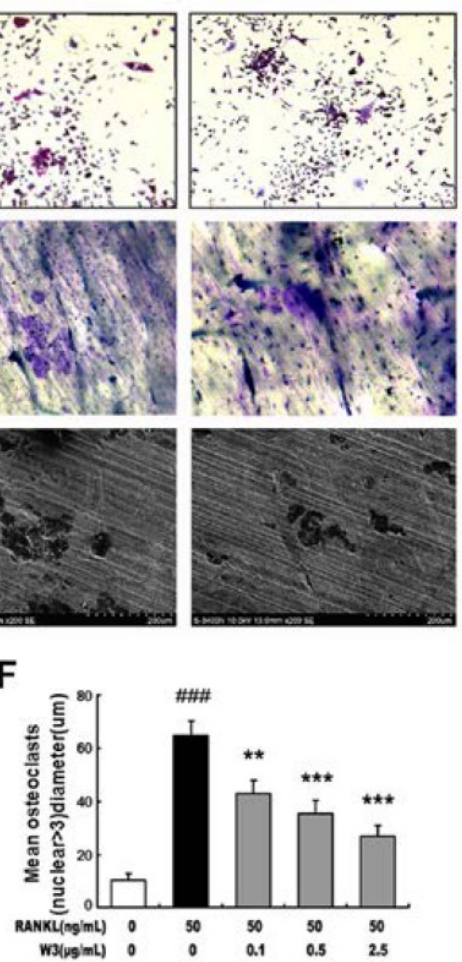

G

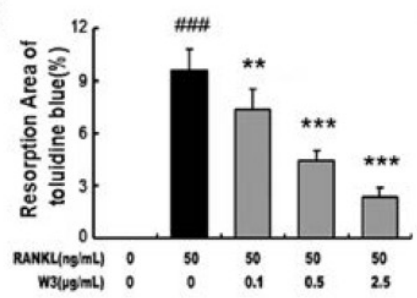

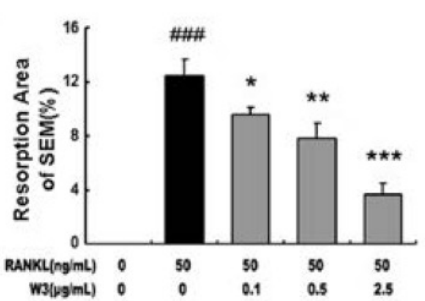

Fig. 3. W3 suppresses osteoclast resorptive activity. RAW 264.7 cells were treated with different concentrations of W3 (0.1, 0.5 and $2.5 \mu \mathrm{g} / \mathrm{mL})$ in the presence of RANKL. After 6 days, performed TRAP staining (A), or cells were removed and the bone slices stained by toluidine blue (B) or by scanning electron microscopy (SEM) to identify resorption pits (C). Quantitative analysis shows the percentage of bovine bone slice surface occupied by resorption lacunae (D-F for TRAP staining, G for toluidine blue staining and $\mathrm{H}$ for SEM). All bar graphs represent mean $\pm \mathrm{SD}$ of three independent experiments. $\# P<0.001$ significantly different from Control. $* P<$ $0.05, * * P<0.01$ and $* * * P<0.001$ significantly different from RANKL only group. 
It is well-known that actin ring structure is a characteristic cytoskeletal feature of functional osteoclasts [12]. The bone resorption function of osteoclasts depends on dynamic regulation of the actin cytoskeleton. Therefore, we next examined whether W3 affects actin ring structure of osteoclasts. Double-stained with phallotoxins and Hoechst 33258 to allow for visualization of the cytoskeleton and nuclei were performed on OCLs respectively. As shown in Fig. 4, F-actin was arranged into a ring-like structure (actin ring) at the cell periphery when mature osteoclasts were induced from RAW 264.7 cells by RANKL on tissue culture plates. Treatment of with W3 caused both shrinkage of osteoclasts and disruption of actin ring structure in a dose-dependent manner.

The effects of W3 on osteoclast formation were further confirmed by primary BMMs. W3 inhibited the multinucleated osteoclast formation induced by RANKL in BMMs (Fig 5 A and B). Meanwhile, there was no obvious cytotoxicity of W3 on BMMs in the presence or absence of RANKL (Fig 5 C).
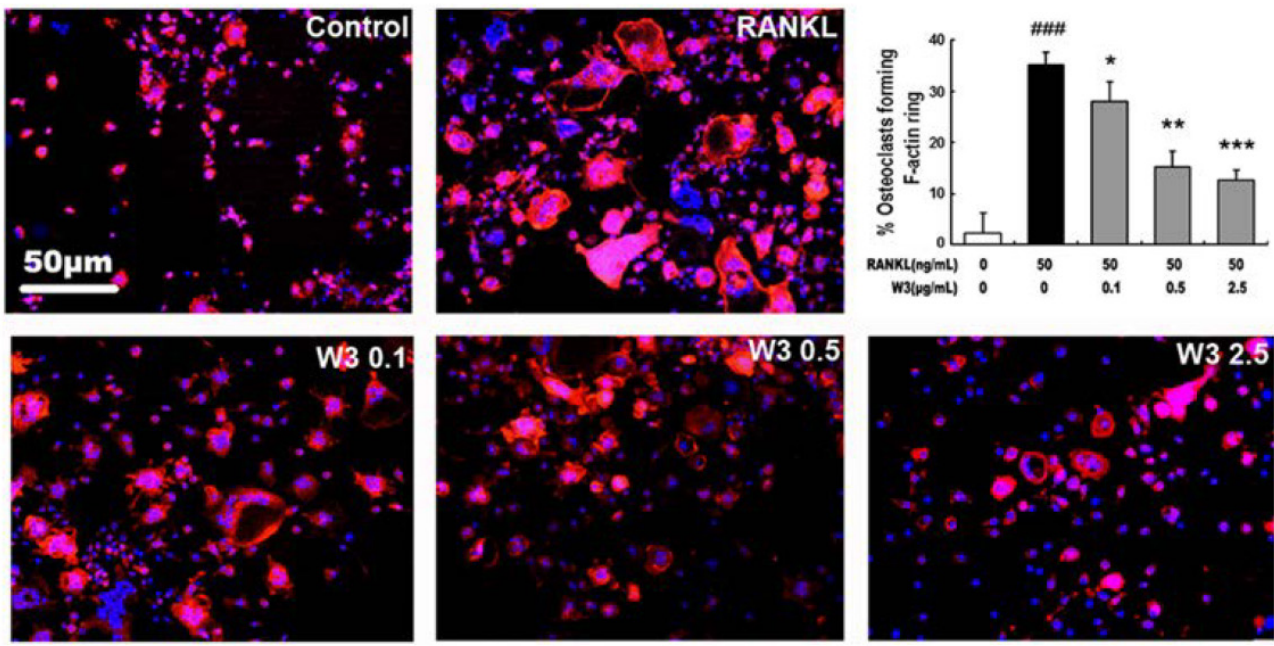

Fig. 4. W3 inhibits the F-actin rings formation of osteoclast. RAW 264.7 cells were cultured in the presence of RANKL with different concentrations of W3 ( $0.1,0.5$ and $2.5 \mu \mathrm{g} / \mathrm{mL}$ ). After 6 days, double stained with Honest 33258 (nuclear staining) and rhodamine-phalloidin (F-actin structure) and visualized by fluorescence microscopy. Quantitative analysis shows percentage of osteoclasts forming F-actin rings. All bar graphs represent mean \pm SD of three independent experiments. \#\# $<0.001$ significantly different from Control. $* P<0.05, * * P<0.01$ and $* * * P<0.001$ significantly different from RANKL only group.

A

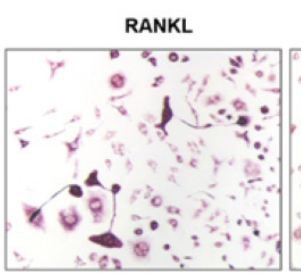

W3 $0.1 \mathrm{ug} / \mathrm{mL}$

W3 $0.5 \mathrm{ug} / \mathrm{mL}$

W3 $2.5 \mathrm{ug} / \mathrm{mL}$

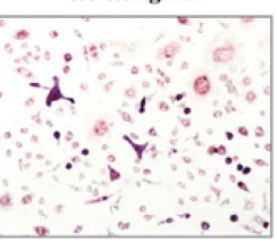

B
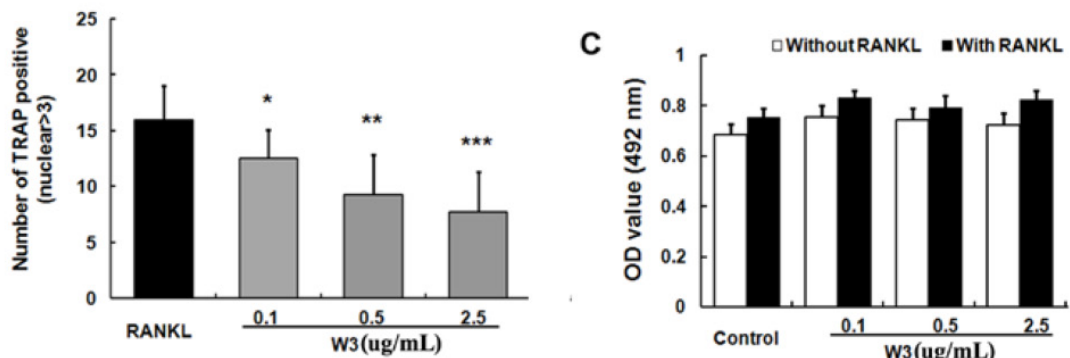

Fig. 5. W3 inhibits RANKL-induced osteoclast differentiation from BMM. BMMs were cultured in the presence of RANKL with different concentrations of W3 (0.1, 0.5 and $2.5 \mu \mathrm{g} / \mathrm{mL}$ ). Six days post-culture, cells were fixed with $4 \%$ paraformadehyde followed by TRAP staining (A). And quantitative analysis of the mean number of TRAP-positive OCLs was performed in this system (B). Cell viability was determined by 3-(4,5-dimethyl-2-thiazolyl)-2,5-diphenyl-2H-tetrazolium bromide (MTS) method when treated with W3 for $48 \mathrm{~h}(\mathrm{C})$. All bar graphs represent mean $\pm \mathrm{SD}$ of three independent experiments. $* P<0.05$, $* * P<0.01$ and $* * * P<0.001$ significantly different from RANKL only group. 


\section{W3 Inhibits RANKL-induced TRAF6 expres- sion}

The trimerization and activation of signaling adaptor molecule TRAF6 followed by RANKL binding with its receptor RANK is an essential initiating step during osteoclast differentiation [13]. So we analyzed the possible action of W3 on TRAF6 protein expression. As shown in Fig. 6 A, RANKL obviously up-regulated the expression of TRAF6 in a time-dependent manner, which reached peak accumulation at $24 \mathrm{~h}$. Therefore, in this study, we explore the effects of W3 on TRAF6 protein expression induced by RANKL for $24 \mathrm{~h}$. Pre-treatment with W3 $(0.1, \quad 0.5$ and $2.5 \mu \mathrm{g} / \mathrm{mL})$ markedly inhibited RANKL-stimulated expression of TRAF6 in a dose-dependent manner (Fig. 6B).

\section{W3 inhibits RANKL-induced MAPKs phos- phorylation}

As the downstream of the TRAF6 signaling complexes, MAPKs (mainly including ERK, JNK and p38 MAPK) play an important role in RANKL-induced osteoclast differentiation. To further explore pathways by which W3 regulates osteoclast differentiation and function, the effect of W3 on RANKL-induced ERK, JNK and p38 phosphorylation was examined in RAW 264.7 cells by western blot. As shown in Fig. 7, RANKL strongly activated ERK, JNK and p38 phosphorylation. When pretreated the cells with various doses of W3 for $2 \mathrm{~h}$, we found that the activation of these signaling pathways was inhibited

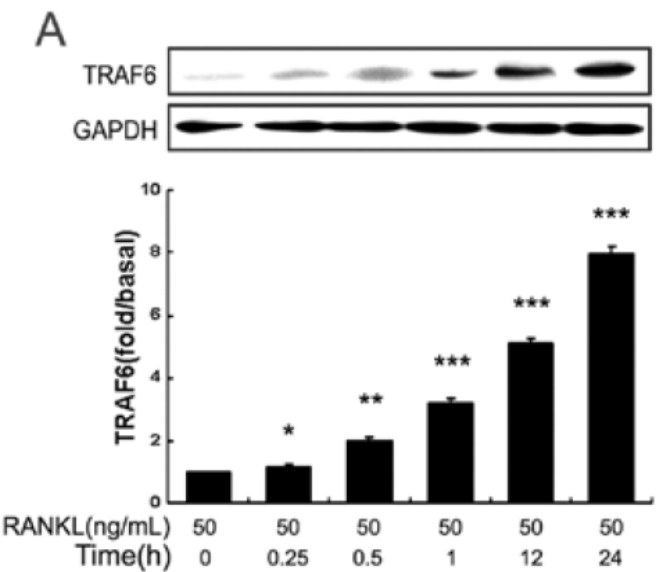

by W3, most effectively for JNK, less for p38, and least for ERK, which indicated that the mechanism for the anti-osteoclast differentiation effects of W3 involved the inhibition of MAPKs.

\section{Involvement of NF- $\kappa B$ signaling pathway in effects of $\mathrm{W} 3$ on the osteoclast differentiation}

RANKL-induced NF-кB activation is essential in initiating osteoclast differentiation. Given that W3 inhibited osteoclast differentiation, we were intrigued to determine if NF- $\mathrm{KB}$ contributes to the function of W3. RAW 264.7 cells were pretreated with W3 followed by RANKL induction, then analyzing the activation of the NF- $\mathrm{kB}$ signaling pathways. As shown in Fig 8A, 30 min of RANKL treatment alone resulted in an increase of IkB-a phosphorylation. Treatment with W3 $(0.1,0.5$ and $2.5 \mu \mathrm{g} / \mathrm{mL}) 2 \mathrm{~h}$ prior to RANKL stimulation, resulted in a significant reduction in IкB-a phosphorylation. In addition, the NF-кB DNA binding activity was investigated in the activated RAW 264.7 cells. As shown in Fig. 8B, compared with that on unstimulated control, RAW 264.7 cells stimulated by RANKL showed a marked increase in NF-kB nuclear protein DNA-binding activity. Pretreatment of cells with various concentrations of W3 suppressed the activation of NF- $\mathrm{kB}$ binding to its consensus DNA sequences. To exclude the non-specificity binding of the NF- $\mathrm{KB}$ nuclear protein-DNA, 200-fold excess of unlabeled NF-kB probe was added in the system. As shown in the Fig. $8 \mathrm{~B}$, there was no NF-kB DNA binding band with unlabeled probe.

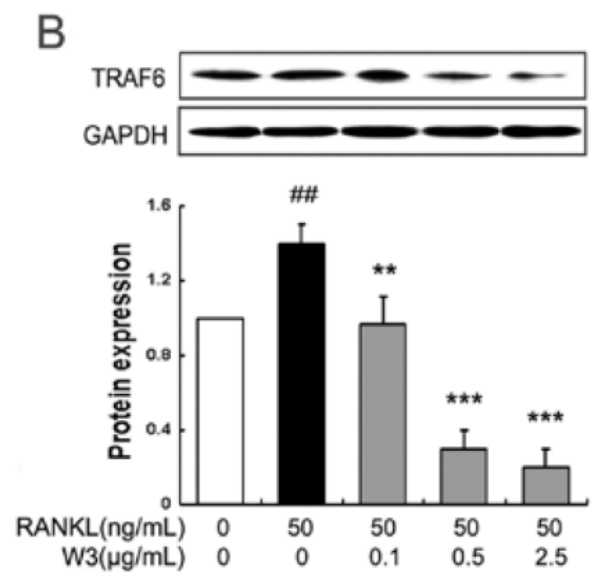

Fig. 6. W3 inhibits the expression of TRAF6 in RAW 264.7 cells treated with RANKL. (A) Time course of the expression of TRAF6 in RANKL-treated RAW 264.7 cells. Cells were treated with RANKL $(50 \mathrm{ng} / \mathrm{mL})$ at various time points as indicated. Expression of TRAF6 was determined by Western blot analysis. (B) RAW 264.7 cells were pretreated with W3 or solvent for $2 \mathrm{~h}$, and then treated with RANKL for $24 \mathrm{~h}$. The cell extracts were subjected to Western blot analysis. Relative amounts of each protein were determined by densitometric analysis. One of three experiments with similar results is shown. Data are represented as the mean \pm SD. \#\#P<0.001 significantly different from control. $* P<0.05$, $* * P<0.01$ and $* * * P<0.001$ significantly different from RANKL only group. 


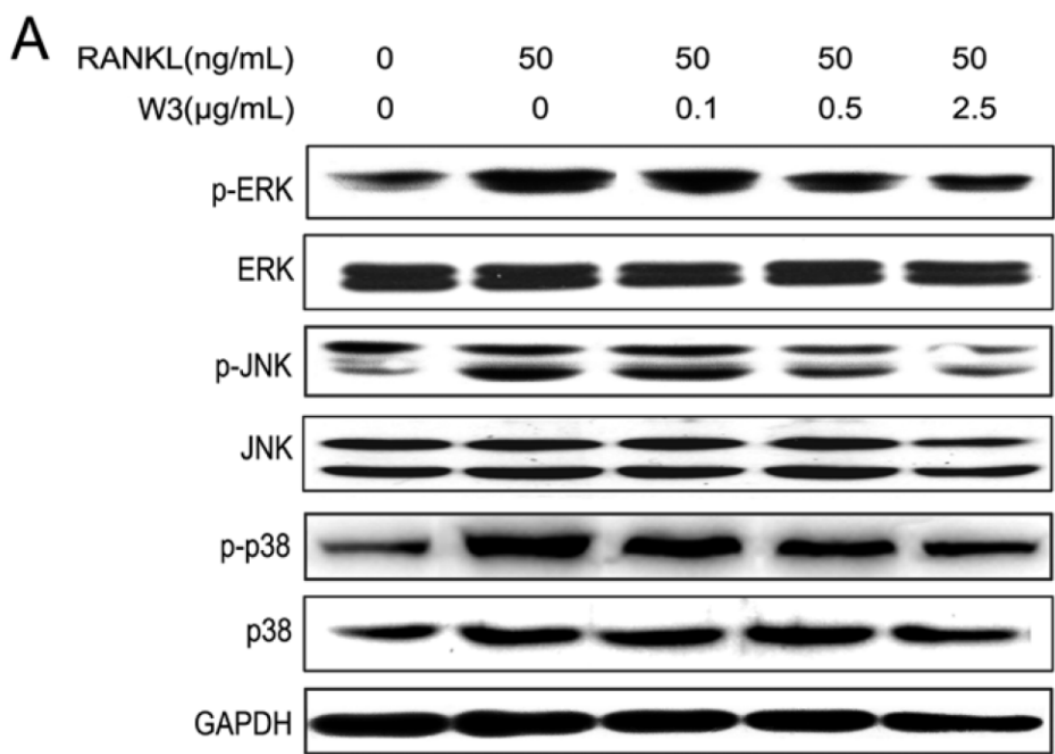

B

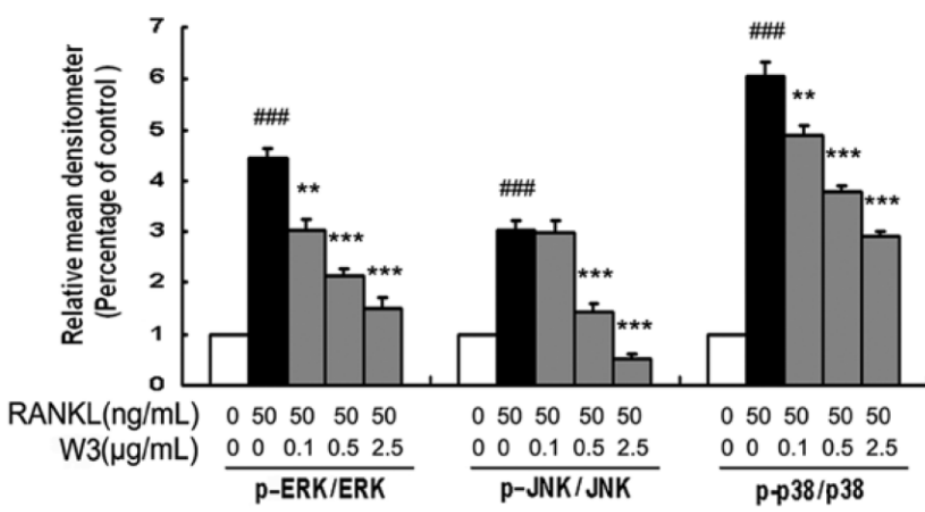

Fig. 7. W3 suppresses MAPKs activation in RAW 264.7 cells induced by RANKL. (A) RAW 264.7 cells were pre-incubated with or without of W3 for $2 \mathrm{~h}$, following treatment with $50 \mathrm{ng} / \mathrm{mL}$ RANKL for $30 \mathrm{~min}$. Total protein was extracted and subjected to Western blot analysis. (B) Relative amounts of each protein were determined by densitometric analysis. One of three experiments with similar results is shown. Data are represented as the mean \pm SD. \#\#P<0.001 significantly different from control. $* P<0.05, * * P<0.01$ and $* * * P<0.001$ significantly different from RANKL only group.

A

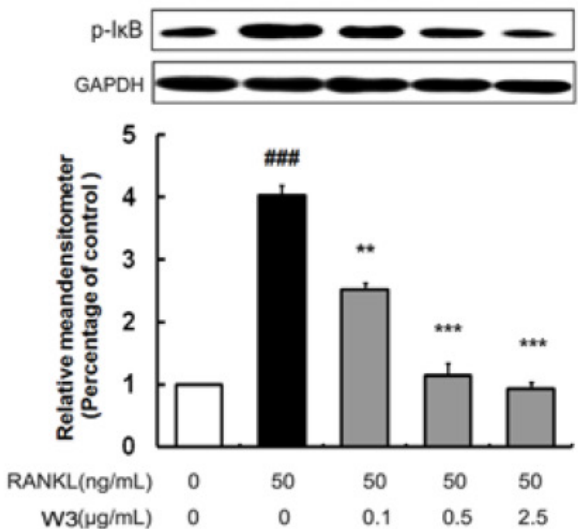

B

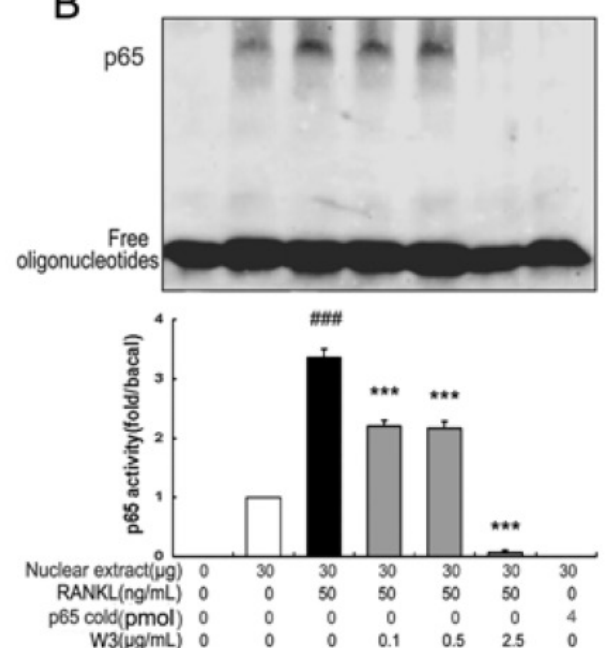

Fig. 8. W3 suppresses NF-kB activation in RANKL-induced RAW 264.7 cells. (A) Confluent RAW 264.7 cells were pre-treated with various concentrations of W3 $(0.1-2.5 \mu \mathrm{g} / \mathrm{mL})$ or vehicle for $2 \mathrm{~h}$ followed by RANKL $(50 \mathrm{ng} / \mathrm{mL})$ for $0.5 \mathrm{~h}$. The harvested cells from triplicate tests were subjected to western blot analysis for p-lkB as described in the text. (B) Confluent RAW 264.7 cells were treated as described above, cells were analyzed for detection of DNA binding of NF-kB by EMSA. Relative NF-kB activity was calculated by densitometric analysis. Data represent the mean $\pm \mathrm{SD}$ of three measurements. \#\#P<0.001 significantly different from basal. $* P<0.05$, and $* * * P<0.001$ significantly different from RANKL only group. 


\section{W3 Down-regulates RANKL-induced expres- sions of NFATcl and c-Fos}

NFATc1 and c-Fos are identified as two of the most important osteoclasts specific transcription factors after RANKL binding to RANK [14]. As shown in Fig. 9A and B, RAW 264.7 cells induced by RANKL for $24 \mathrm{~h}$ markedly increased the expressions of both c-Fos and NFATc1. However, W3 (0.1, 0.5 and 2.5 $\mu \mathrm{g} / \mathrm{mL})$ treatments significantly inhibited the expression of c-Fos and NFATc1.

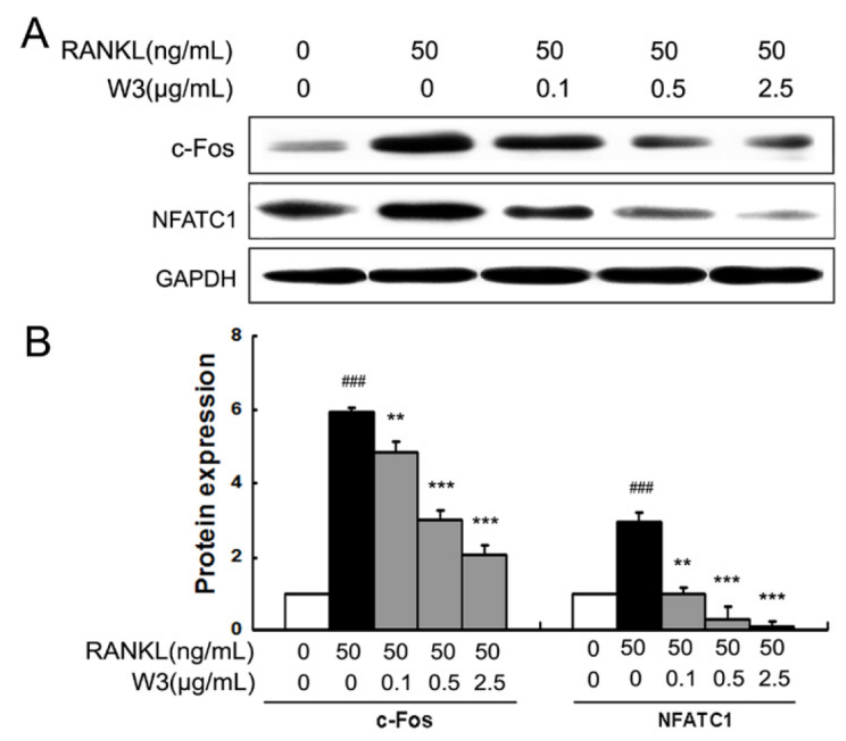

Fig. 9. W3 down-regulates the expression of NFTAcl and c-Fos in RANKL-treated RAW 264.7 cells. (A) RAW 264.7 cells were treated with indicated concentrations of W3 or solvent for $2 \mathrm{~h}$, followed by RANKL for $24 \mathrm{~h}$. The harvested cells from triplicate tests were subjected to Western blot analysis. (B) Relative amounts of each protein were determined by densitometric analysis. Data represent the mean $\pm S D$ of three measurements. $\ldots$ \#\# 0.001 significantly different from basal. $* P<0.05$, and $* * * P<0.001$ significantly different from RANKL only group.

\section{Discussion}

The root of A. flaccida has long been used for the treatment of arthritis and traumatic injury in folk medicine of China [15]. Although diverse mechanisms have been discussed, only a few direct molecular targets have been identified $[4,16]$. In particular, targets responsible for the anti-arthritis effects of A. flaccida are still elusive. Research over the last decade reveals a prominent role of osteoclast in the pathogenesis of RA, especially excessive bone resorption by osteoclasts within inflamed joints is the most specific hallmark of RA [17, 18]. Accordingly, inhibitors of osteoclast possess potential therapeutic action of bone and joint destruction in RA. From this, we speculated that an interference with osteoclast might contribute to the anti-arthritis activity of $A$. flaccida. In fact, our study confirms saponin W3 as an active ingredient of $A$. flaccida, selectively suppressing osteoclast differentiation and bone resorption activity via down-regulation of the TRAF6 level, inhibition of MAPKs and NF-KB activation, and subsequent decreased expression of c-Fos and NFATc1, which summarized in Fig. 10. These data provide the mechanistic insight, at least in part, for the protective effect of A. flaccida against lytic bone diseases.

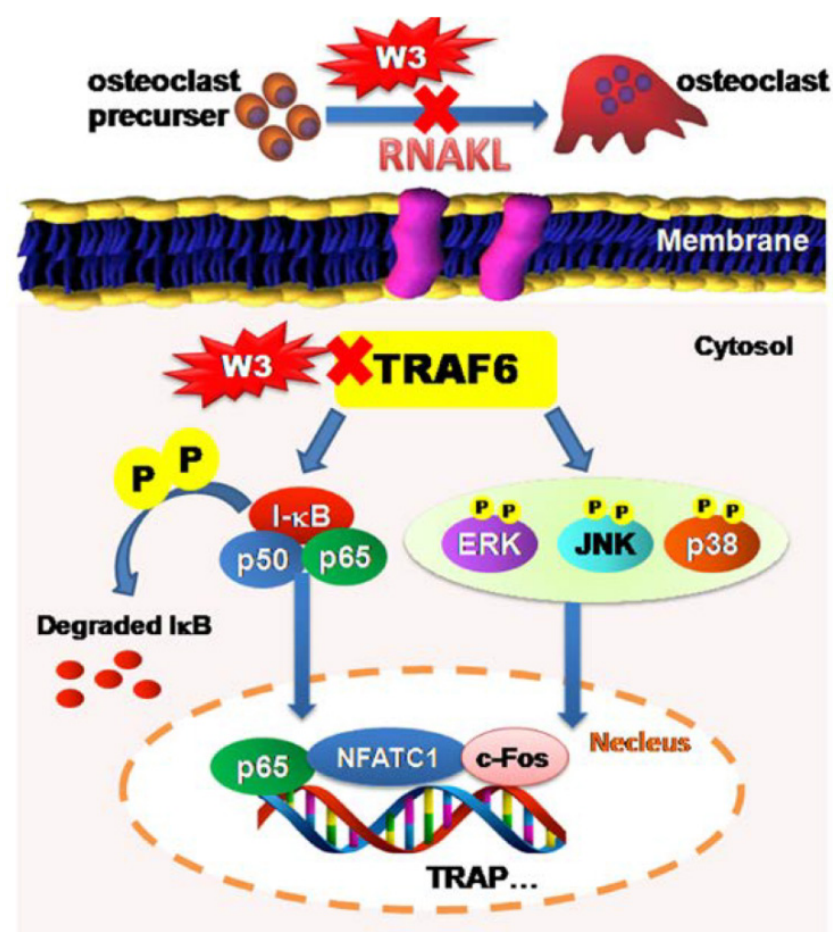

Fig. 10. Schematic illustration showing putative-signaling pathways involved in osteoclast differentiation regulated by W3. The figure summarizes the results presented in this study. Red $X$ mark indicates that an inhibition or a down regulation in osteoclast was observed.

Osteoclasts differentiation from macrophages can be induced by RANKL, a key factor that also controls the function and survival of mature osteoclasts [19]. In this study, both RAW 264.7 and BMMs cells were induced by RANKL to be differentiated as osteoclast. Previous studies have shown that saponins are the characteristic components and also the main active ingredients of A. flaccida [3]. Among them, triterpenoid saponin W3 is the most abundant saponin at the concentration up to $51.8 \%$ in TS, and can also be synthesized [5]. Interestingly, we found that W3 was apparently the most effective ingredient among all the tested triterpenoid saponins to attenuate osteoclastic bone resorption as analyzed by the bone resorption pit assay. It has been well demonstrated that the formation of bone resorption closely associated with the differentiation of osteoclast. Further investigation revealed that W3 suppressed osteoclastic bone resorption through inhibiting osteoclast 
differeantiation. Our results obtained from this study implicate that W3 has the potential to be a drug candidate for treating bone associated diseases and provide insights into the molecular mechanism by which W3 impacts osteoclast differentiation induced by RANKL.

RANKL bind to its receptor RANK on the surface of osteoclast precursors to induce differentiation [20]. The binding of RANKL with its receptor RANK initiates a signaling cascade involving the activation of TRAF6, a signaling adaptor molecule in osteoclast precursors leads to the activation of the MAPKs and NF-kB pathways. Studies have revealed that MAPKs as one of the important downstream pathways in TRAF6 mediated- osteoclast differentiation [21-23]. It is well known that there are three main subfamilies of MAPKs as JNK, p38 and ERK1/2 MAPK. Previous reports showed that specific inhibitors for JNK, p38 and ERK1/2 MAPK could inhibit the osteoclast differentiation induced by RANKL, suggesting that MAPKs contribute to the RANKL-induced osteoclast differentiation and bone-resorbing activity [24-27]. Our result showed that W3 significantly inhibited TRAF6 expression, subsequently suppressed the phosphorylation of ERK 1/2, JNK, and p38 MAPK, implicating that W3 impacts osteoclast differerntiation and bone-resorbing activity through inhibiting the activation of the MAPK pathway. In addition, it has been well established that NF-kB signaling pathway also plays an indispensable role in osteoclast differentiation as another downstream pathway of TRAF6 signaling [28]. The NF-кB signaling pathway can be activated by either an IкB dependent or independent way. The classical NF-kB pathway involves the activation of the IKK complex, resulting in phosphorylation of IkBa, which is subsequently degraded through the ubiquitin-dependent proteosome system [13]. In the alternative IKB-independent pathway, direct phosphorylation of NF-kB subunit p65 by IKK also modulates NF-אB transcription activity [29]. Previous studies showed that the defects of osteoclast differentiation and severe osteopetrosis in NF- $\mathrm{kB}$ knockout mice [30]. As shown in Fig. 8, W3 inhibited NF-kB activation in RANKL-induced RAW 264.7 cells as indicated by decreased p-IкBa and the DNA binding activity of p65. Collectively, our data suggest that W3 inhibits osteoclast differentiation and function through the suppression of both MAPKs and NF-kB pathways.

Multiple osteoclastogenic transcription factors are believed to play a vital role in the function of the formation of mature and functionally active osteoclasts. Studies have revealed that c-Fos and NFATc1 mediate osteoclast differentiation. NFATc1 is a downstream target of c-Fos in regulating osteoclast differentiation by RANKL stimulation. Mice lacking c-Fos develop osteopetrosis as a result of a complete ablation of osteoclast formation. Previous studies reported that NFATc1-deficient embryonic stem cells do not form mature osteoclasts induced by RANKL. Moreover, overexpression of ectopic ca-NFATc1 in BMMs appropriately induces osteoclast differentiation even in the absence of RANKL [31, 32]. In our study, exposure of RAW 264.7 cells to RANKL resulted in the increased expression of both c-Fos and NFATc1, leading to osteoclast formation. However, W3 treatment significantly suppressed the expression of c-Fos and NFATc1, suggesting its role in osteoclast differentiation.

Taken together, our results reveal that W3 suppressed RANKL-induced osteoclast differentiation via the down-regulation of the TRAF6 level, inhibition of MAPKs and NF- $\mathrm{kB}$ activation, and subsequently decreased expression of c-Fos and NFATc1, suggesting that $\mathrm{W} 3$, one of the major ingredients of the traditional herbal remedy A. flaccid has the potential to be a drug candidate for treating lytic bone diseases such as arthritis although further in vivo investigation is needed.

\section{Acknowledgments}

This study was supported by the major projects of national science and technology (2011ZX09101006-03, 2013ZX09301307), natural science foundation of Beijing (7112096), and the National Natural Science Foundation of China (81274167).

\section{Abbreviations}

a-MEM: alpha-modified eagles medium, ELISA: enzyme linked immunosorbent assay, EMSA: electrophoretic mobility shift assay, ERK1/2: extracellular signal-regulated kinase 1/2, FBS: fetal bovine serum, JNK: c-Jun N-terminal kinase, MAPKs: mitogen-activated protein kinases, MTS: 3-(4,5-dimethyl-2-thiazolyl)-2,5-diphenyl-2H-tetrazoli um bromide, NFATc1: nuclear factor of activated T cells, NF-kB: nuclear factor-kb, OCL: osteoclast-like, RANKL: Receptor activator of nuclear factor kappa-B ligand, TRAF6: tumor necrosis factor receptor-associated factor 6, TRAP: tartrate-resistant acid phosphatase, TS: total saponins.

\section{Competing Interests}

The authors have declared that no competing interest exists.

\section{References}

1. Boyle WJ, Simonet WS, Lacey DL: Osteoclast differentiation and activation. Nature 2003, 423(6937):337-342. 
2. Tanaka Y, Nakayamada S, Okada Y: Osteoblasts and osteoclasts in bone remodeling and inflammation. Current drug targets Inflammation and allergy 2005, 4(3):325-328.

3. Han LT, Li J, Huang F, Yu SG, Fang NB: Triterpenoid saponins from Anemone flaccida induce apoptosis activity in HeLa cells. Journal of Asian natural products research 2009, 11(2):122-127.

4. Bing F, Zhang G, Deng C, Gong Y: Immune mechanisms of Wugong Sanqi extract against CIA in mouse. Chinese Journal of Immunology 2008, 24(8):716-720.

5. Cheng S, Du Y, Bing F, Zhang G: Synthesis of flaccidoside II, a bidesmosidic triterpene saponin isolated from Chinese folk medicine Di Wu. Carbohydrate research 2008, 343(3):462-469.

6. Han LT, Fang Y, Li MM, Yang HB, Huang F: The Antitumor Effects of Triterpenoid Saponins from the Anemone flaccida and the Underlying Mechanism. Evidence-based complementary and alternative medicine : eCAM 2013, 2013:517931.

7. Liao Y: Application of chemical synthesis technology in the development of new Chinese Drugs. Hubei University of traditional Chinese Medicine; 2010.

8. Cully M: Rheumatoid arthritis: Uncoupling bone and cartilage destruction. Nature reviews Rheumatology 2013, 9(8):446.

9. Kong X, Liu C, Zhang C, Zhao J, Wang J, Wan H, Zhu H, Zhang P, Chen W, Xiao $\mathrm{Y}$ et al: The suppressive effects of Saposhnikovia divaricata (Fangfeng) chromone extract on rheumatoid arthritis via inhibition of nuclear factor-kappaB and mitogen activated proteinkinases activation on collagen-induced arthritis model. Journal of ethnopharmacology 2013, 148(3):842-850.

10. Hsu H, Lacey DL, Dunstan CR, Solovyev I, Colombero A, Timms E, Tan HL, Elliott G, Kelley MJ, Sarosi I et al: Tumor necrosis factor receptor family member RANK mediates osteoclast differentiation and activation induced by osteoprotegerin ligand. Proceedings of the National Academy of Sciences of the United States of America 1999, 96(7):3540-3545.

11. Huang QQ, Pope RM: The role of toll-like receptors in rheumatoid arthritis. Current rheumatology reports 2009, 11(5):357-364.

12. Griese JJ, R PJ, Schwarzinger S, Dobbek H: Xenobiotic reductase A in the degradation of quinoline by Pseudomonas putida 86: physiological function, structure and mechanism of 8-hydroxycoumarin reduction. Journal of molecular biology 2006, 361(1):140-152

13. Asagiri $\mathrm{M}$, Takayanagi $\mathrm{H}$ : The molecular understanding of osteoclast differentiation. Bone 2007, 40(2):251-264

14. Cheng B, Li J, Du J, Lv X, Weng L, Ling C: Ginsenoside Rb1 inhibits osteoclastogenesis by modulating NF-kappaB and MAPKs pathways. Food and chemical toxicology : an international journal published for the British Industrial Biological Research Association 2012, 50(5):1610-1615.

15. Medicine SAoTC: Chinese Materia Medica, vol. 7, 1st edn. Shanghai: Shanghai science and Technology Press; 1999.

16. Bing F, Zhang G: The national five kind of new medicine--rhizome of Anemone Flaccid Fengshi'an capsule. Hubei Journal of Traditional Chinese Medicine 2005, 27(1):48-49.

17. Tanaka S, Nakamura K, Oda H: The osteoclast: a potential therapeutic target of bone and joint destruction in rheumatoid arthritis. Modern rheumatology/the Japan Rheumatism Association 2001, 11(3):177-183.

18. Hirayama T, Danks L, Sabokbar A, Athanasou NA: Osteoclast formation and activity in the pathogenesis of osteoporosis in rheumatoid arthritis. Rheumatology (Oxford) 2002, 41(11):1232-1239.

19. Takayanagi H, Kim S, Matsuo K, Suzuki H, Suzuki T, Sato K, Yokochi T, Oda $\mathrm{H}$, Nakamura $\mathrm{K}$, Ida $\mathrm{N}$ et al: RANKL maintains bone homeostasis through c-Fos-dependent induction of interferon-beta. Nature 2002, 416(6882):744-749.

20. Lacey DL, Timms E, Tan HL, Kelley MJ, Dunstan CR, Burgess T, Elliott R, Colombero A, Elliott G, Scully S et al: Osteoprotegerin ligand is a cytokine that regulates osteoclast differentiation and activation. Cell 1998, 93(2):165-176.

21. Huh JE, Jung IT, Choi J, Baek YH, Lee JD, Park DS, Choi DY: The natural flavonoid galangin inhibits osteoclastic bone destruction and osteoclastogenesis by suppressing NF-kappaB in collagen-induced arthritis and bone marrow-derived macrophages. European journal of pharmacology 2013, 698(1-3):57-66

22. Li C, Yang Z, Li Z, Ma Y, Zhang L, Zheng C, Qiu W, Wu X, Wang X, Li H et al: Maslinic acid suppresses osteoclastogenesis and prevents ovariectomy-induced bone loss by regulating RANKL-mediated NF-kappaB and MAPK signaling pathways. Journal of bone and mineral research : the official journal of the American Society for Bone and Mineral Research 2011, 26(3):644-656.

23. Zhai ZJ, Li HW, Liu GW, Qu XH, Tian B, Yan W, Lin Z, Tang TT, Qin A, Dai KR: Andrographolide suppresses RANKL-induced osteoclastogenesis in vitro and prevents inflammatory bone loss in vivo. British journal of pharmacology 2014, 171(3):663-675

24. Chen D, Guo Y, Mao X, Zhang X: Inhibition of p38 mitogen-activated protein kinase down-regulates the inflammatory osteolysis response to titanium particles in a murine osteolysis model. Inflammation 2012, 35(6):1798-1806.

25. Tao $H$, Okamoto $M$, Nishikawa $M$, Yoshikawa $H$, Myoui A: P38 mitogen-activated protein kinase inhibitor, FR167653, inhibits parathyroid hormone related protein-induced osteoclastogenesis and bone resorption. PloS one 2011, 6(8):e23199.

26. Ikeda F, Matsubara T, Tsurukai T, Hata K, Nishimura R, Yoneda T: JNK/c-Jun signaling mediates an anti-apoptotic effect of RANKL in osteoclasts. Journal of bone and mineral research : the official journal of the American Society for Bone and Mineral Research 2008, 23(6):907-914.
27. Yamanaka Y, Clohisy JC, Ito H, Matsuno T, Abu-Amer Y: Blockade of JNK and NFAT pathways attenuates orthopedic particle-stimulated osteoclastogenesis of human osteoclast precursors and murine calvarial osteolysis. Journal of orthopaedic research : official publication of the Orthopaedic Research Society 2013, 31(1):67-72.

28. Takayanagi H: Osteoimmunology: shared mechanisms and crosstalk between the immune and bone systems. Nature reviews Immunology 2007, 7(4):292-304.

29. Sakurai $H$, Chiba $H$, Miyoshi $H$, Sugita $T$, Toriumi W: IkappaB kinases phosphorylate NF-kappaB p65 subunit on serine 536 in the transactivation domain. The Journal of biological chemistry 1999, 274(43):30353-30356.

30. Leibbrandt A, Penninger JM: RANKL/RANK as key factors for osteoclast development and bone loss in arthropathies. Advances in experimental medicine and biology 2009, 649:100-113.

31. Takayanagi $H$, Kim $S$, Koga $T$, Nishina $H$, Isshiki M, Yoshida $H$, Saiura A, Isobe $\mathrm{M}$, Yokochi $\mathrm{T}$, Inoue $\mathrm{J}$ et al: Induction and activation of the transcription factor NFATc1 (NFAT2) integrate RANKL signaling in terminal differentiation of osteoclasts. Developmental cell 2002, 3(6):889-901.

32. Lee SH, Kim T, Jeong D, Kim N, Choi Y: The tec family tyrosine kinase Btk Regulates RANKL-induced osteoclast maturation. The Journal of biological chemistry 2008, 283(17):11526-11534. 